\title{
Thermo-chemical analysis and modeling of combustion of waste pyrolysis gaseous products
}

\author{
Alexey Demin 1,*, Grigorii Pavlov², and Mansur Khasiyatullov \\ ${ }^{1}$ Kazan State Power Engineering University, 420066 Kazan, Krasnoselskaya Str. 51, Russia \\ ${ }^{2}$ Kazan National Research Technical University named after A.N. Tupolev - KAI, 420111 Kazan, K. Marx Str. 10, Russia \\ ${ }^{3}$ Management Company "Integrated EnergoDevelopment- Holding (IED - Holding)", 420036 Kazan, Voskhod Str. 45a, Russia
}

\begin{abstract}
The results of the study of joint pyrolysis of various types of waste (municipal solid waste, plastic waste, etc.) are presented. Preliminarily crushed and dried wastes were fed into the pyrolysis chamber of the model experimental setup. Thermal energy required for heating raw materials and carrying out their thermal destruction was obtained by burning a part of the pyrolysis gases. The rest of these gases were removed from the pyrolysis chamber and cooled. The temperature in the pyrolysis zone was about 650 ${ }^{\circ} \mathrm{C}$. Plant productivity was up to $500 \mathrm{~kg} / \mathrm{h}$. The target product was the liquid phase, which is a mixture of hydrocarbon compounds. When organizing the processes, the yield of solid carbon residue was minimized. The obtained mass ratio of the final gas/liquid products was approximately equal to $1 / 6$. Experimental results of the analysis of the chemical composition of the gas and liquid fractions are presented. The results of modeling the combustion of pyrolysis products at different amounts of supplied air are also shown. The operating parameters at which the optimum temperature level in the pyrolysis zone is maintained are numerically determined and recommended.
\end{abstract}

\section{Introduction}

Waste disposal is one of the global challenges of our time, as constantly increasing waste amounts have severely negative impacts on the environment. The hazardous environmental issues include alienation of territories necessary for waste disposal, pollution of atmospheric air, land resources, surface and ground waters, etc. An obvious solution to these problems is the involvement of waste into repeated economic circulation: processing, regeneration, recovery and recycling. Improving efficiency and introducing energy and resource-saving waste disposal technologies are important scientific and technical tasks. This article examines the carbon-containing waste that is a part of typical municipal waste: plastic, polyethylene, packaging materials, etc.

Currently, waste is used to obtain heat and electricity, as well as for secondary raw materials. The most promising methods include thermal processing: pyrolysis, gasification, and combustion. Waste combustion allows to obtain thermal energy, which is then converted into electrical energy. Importantly, waste recycling results in the reduced consumption of traditional fuels, including natural gas, oil products, and coal. During gasification of waste, the main product is a mixture of combustible gases, i.e., $\mathrm{CO}, \mathrm{CO} 2, \mathrm{H} 2, \mathrm{CH} 4$ and others. In waste pyrolysis, the products include carbonaceous solid residue, a liquid mixture of complex hydrocarbons, and relatively small amounts of a non- condensable gas phase. The quantitative yield of a certain product depends on the heating rate of the starting materials, the residence time in the reaction volume, and the pressure values during the conversion process. The process is carried out at very low oxygen concentrations or in its complete absence at temperatures ranging from $500^{\circ} \mathrm{C}$ to $800^{\circ} \mathrm{C}$. The pyrolysis products have a higher energy value than the starting materials, and when burned, they can provide higher temperatures of the combustion products than when burning the starting material. Liquid pyrolysis products are used not only as an alternative fuel. Their practical importance as raw materials in chemical technologies is obvious.

The main regularities of the processes for the listed methods of thermal waste treatment are well known. Special attention is paid to the experimental and theoretical study of various aspects related to the search for the most efficient organization of processes, mainly depending on the selected processing method, chemical composition and aggregate state of the waste [1-16].

An increase in the yield of gas mixture due to the increased concentration of carbon monoxide is facilitated by the implementation of pyrolysis when supplying carbon dioxide [1]. The work [2] shows a significant effect of the presence of zeolite catalysts on the increased gas yield during pyrolysis. However, when using catalysts, it is necessary to periodically regenerate them. Studies of tire pyrolysis are of great interest $[3,4]$. This technology offers an attractive increase in the yield of liquid hydrocarbons, which are valuable chemical raw

\footnotetext{
* Corresponding author: alexei_demin@mail.ru
} 
materials. Since the need for plastic waste recycling is constantly increasing, the research on plastic pyrolysis processes is also increasing [5-12]. In the pyrolysis of plastic with the use of a catalyst, valuable products are hydrocarbons, which are a part of the oil light fractions [5]. The studies of thermochemical conversion during the joint pyrolysis of various types of waste show the presence of a synergistic effect [13]. The waste mixture has a wider thermal decomposition temperature range. By mixing waste in certain proportions, one can reduce the amount of heat energy required for pyrolysis. Certain difficulties in the design and operation of pyrolysis setups for municipal solid waste are due to the wide variety of waste composition $[14,15,16]$. The complex composition of waste predetermines the need for further search for the most effective ways of organizing pyrolysis processes.

Here, we studied the pyrolysis processes occurring in a model operating setup. The heat energy required for the thermo-chemical conversion of the solid waste mixture was obtained by burning a part of the pyrolysis gases. The combustion of these gases is organized near the outer surface of the pyrolysis chamber. The gases used for this purpose enter the combustion zone through perforations in the pyrolysis chamber. Combustion products do not return to the pyrolysis zone. The main goal of our research was to determine the amount of air required to burn this part of the pyrolysis gases in order to ensure the optimal temperature level in the pyrolysis chamber.

\section{Materials and methods}

The principle of organizing processes in a model setup is shown in Figure 1.

Solid waste is fed into the chamber 11 for preliminary drying. The dried waste is transported to the pyrolysis chamber 1 , where its thermal destruction takes place. Most of the pyrolysis gases are directed to the condenser 12, where the gas phase is transformed into the liquid phase. The liquid phase is the main useful product. A smaller part of the pyrolysis gases from the pyrolysis chamber 1 enters the thermal decomposition chamber 2. Preheated primary air is supplied to the chamber 2 and pyrolysis gases are burned with a lack of oxidant. The heat energy obtained during combustion in the chamber 2 is used to maintain the required temperature level in the pyrolysis chamber 1 . At the exit from the chamber 2, products with an excess of combustible substances are formed. This mixture is directed to the combustion chamber 3, into which secondary air is supplied. The complete combustion of combustible substances takes place in the chamber 3.The combustion chamber 3 is structurally made in conjunction with the mixing chamber 4 . In the chamber 4 , combustion products are mixed with tertiary air. In this chamber, the temperature of the mixture is reduced from $1200{ }^{\circ} \mathrm{C}$ to $550-600^{\circ} \mathrm{C}$. The diluted combustion products from the mixing chamber 4 are fed to the cyclone 5, where solid particles are separated from the gas phase. A small part of the ash residue, which is suspended in the combustion products, is captured by the cyclone 5 and the filter 8 , installed in the gas path. For preliminary drying of the feedstock, heated air obtained in the heat exchanger 6is used. The removal of the cleaned combustion products into the atmosphere occurs with the help of a fan (smoke exhauster) through the exhaust pipe 10.The solid residue from the pyrolysis chamber 1 enters the thermal decomposition chamber 2 , where the carbon residue is burned out. The main part of the ash is removed from the thermal decomposition chamber 2 .

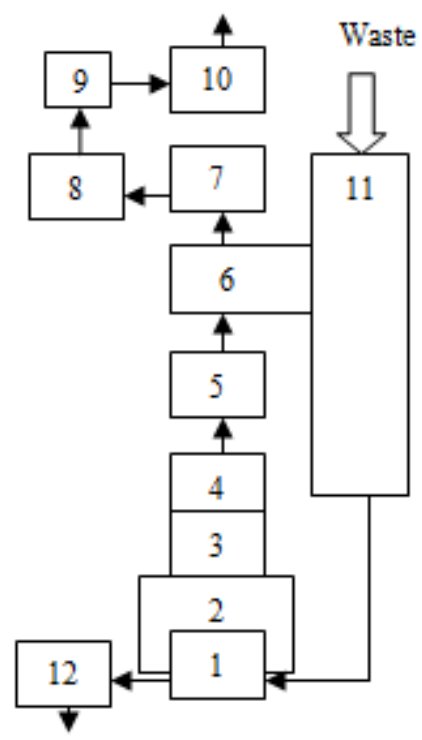

Fig. 1. Schematic view of the model setup. The setup consists of the (1) pyrolysis chamber, (2) thermal decomposition chamber,(3) combustion chamber, (4) mixing chamber, (5) cyclone, (6) heat exchanger, (7) acid gas neutralizer, (8) filter, (9) smoke exhauster (or fan), (10) exhaust pipe, (11) drying chamber, and (12) condenser.

The organic part of the pyrolysis products was studied by the method of sorption of organic substances, followed by the study of the desorbed gas phase on the chromatography-mass spectrometer DSQ II/Focus (Thermo).The sampling of products of thermal decomposition of waste from the investigated zone of the model setup was carried out as follows. Pyrolysis gases were pumpedat a rate of $1 \mathrm{~L} / \mathrm{min}$ for $5 \mathrm{~min}$ through the sorption tubes preconditioned in a flow of dry helium. After that, the sorb containers were delivered to the laboratory. When measuring the composition of gases and conducting analysis, the following measuring instruments, materials and reagents were used: gas chromatograph Focus (Thermo) (capillary column VF$1 \mathrm{~ms}$ - length $30 \mathrm{~m}$, inner diameter $0.25 \mathrm{~mm}$, stationary phase thickness $0.25 \mu \mathrm{m})$; mass-selective detector (DSQII: electron impact ionization, ionization energy 70 $\mathrm{eV}$, noise/signal ratio $\mathrm{m} / \mathrm{z}-272$ ); two-stage thermodesorber TDS-1 with a cooled trap on the Peltier element (maximum desorption temperature $250^{\circ} \mathrm{C}$, operating temperature range of the trap from $25^{\circ} \mathrm{C}$ to $250^{\circ} \mathrm{C}$, trap heating rate $1000^{\circ} \mathrm{C} 1 / \mathrm{min}$ ). The chromatographic parameters were the following: evaporator temperature of $250^{\circ} \mathrm{C}$; linear temperature 
programming from $80^{\circ} \mathrm{C}$ to $280^{\circ} \mathrm{C}$; scanning mode of $50-600 \mathrm{~m} / \mathrm{z}$.

The experimental results were processed according to the following procedure. The sorption tube was placed into the thermal desorber. Air was removed from the tube to avoid incorrect chromatograph data arising from the thermal oxidation of the sorbent or the stationary phase used in gas chromatography. The tube was then heated to desorb organic vapors entering the column in the carrier gas stream. The carrier gas flow was directed towards the diffusion end of the tube, i.e., the marked end of the tube was located at the entrance to the column of the chromatograph. After the thermal desorber switch to the mode ready for injecting the desorbed sample into the evaporator, it switched to the standby mode. The sample was injected automatically when the chromatograph was ready. Peaks in the chromatogram were identified using the NIST MS Search 2.0 mass spectrometric library.

The obtained experimental data on the composition of pyrolysis products were used to determine the amount of air required to burn a part of the pyrolysis gases. Conditions typical for the chemically equilibrium state of products from incomplete combustion were simulated. The methodology and the calculation program presented in [17] were used here.

\section{Results and discussion}

The chemical composition of the condensed phase of pyrolysis products is shown in Table 1 .

Table 1. The chemical composition of the condensed phase of pyrolysis products.

\begin{tabular}{|l|c|}
\hline Chemical compound & Concentration, wt. \% \\
\hline Indene & 17.784 \\
\hline Undecene-1 & 6.248 \\
\hline 3,4-Dimethylstyrene & 1.802 \\
\hline 2-Methylindene & 3.88 \\
\hline 3-Methylindene & 1.648 \\
\hline Dodecene & 3.11 \\
\hline Naphthalene & 50.218 \\
\hline Tridecene & 2.89 \\
\hline 2-Methylnaphthalene & 1.968 \\
\hline 1-Methylnaphthalene & 4.564 \\
\hline Tetradecene & 1.668 \\
\hline Acenaphthylene & 1.628 \\
\hline Pentadecene & 1.498 \\
\hline Acenaphthene & 0.546 \\
\hline Hexadecene & 0.542 \\
\hline
\end{tabular}

In the condensed phase of pyrolysis products, polycyclic aromatic hydrocarbons were present in the greatest amounts $(\sim 82 \mathrm{wt} . \%)$. Unsaturated hydrocarbon compounds with double bonds were in smaller amounts (alkenes $\sim 16$ wt. \%). The content of arenes was less than 2 wt. $\%$.

The chemical composition of the mixture of noncondensable gases is shown in Table 2.
Table 2. The chemical composition of the mixture of noncondensable gases.

\begin{tabular}{|l|c|}
\hline Chemical compound & Concentration, wt. \% \\
\hline Carbon monoxide & 7.37 \\
\hline Methane & 15.22 \\
\hline Carbon dioxide & 11.73 \\
\hline Acetylene & 0.20 \\
\hline Ethylene & 29.67 \\
\hline Ethane & 9.21 \\
\hline Water & 0.95 \\
\hline Propylene & 14.53 \\
\hline Chloromethane & 0.12 \\
\hline Propadiene & 0.12 \\
\hline Propane & 0.66 \\
\hline Propine & 0.23 \\
\hline Vinylchloride & 0.11 \\
\hline Vinylacetylene & 0.08 \\
\hline 2-Methylpropene & 2.45 \\
\hline 1.3-Butadiene & 4.88 \\
\hline Butylene-1 & 0.48 \\
\hline Butane & 0.11 \\
\hline 2-Butane & 0.35 \\
\hline 1.2-Butadiene & 0.01 \\
\hline Pentene-2 & 0.02 \\
\hline Cyclopentene & 0.05 \\
\hline Cyclopentadiene & 0.56 \\
\hline Pentadiene & 0.40 \\
\hline Pentane & 0.24 \\
\hline Benzene & 0.14 \\
\hline Hexane & 0.07 \\
\hline & \\
\hline & \\
\hline
\end{tabular}

A wider range of chemicals was observed in the noncondensable gas phase. Alkenes ( 47.5 wt. \%) and alkanes $(\sim 25.5$ wt. $\%)$ were in the largest amounts. The alkadienes content did not exceed $6 \mathrm{wt}$. \%.The total content of alkynes, cycloalkanes, unsaturated hydrocarbons with double and triple bonds, halogenated hydrocarbons and arenes (benzene) did not exceed 1.5 wt. \%.

In the composition of pyrolysis products combusted to provide thermal energy for pyrolysis processes, the amount of condensed and non-condensable gases was $\sim 84$ wt. $\%$ and $\sim 16$ wt. $\%$, respectively. The amount of air required for complete combustion of combustible gases was $13.22 \mathrm{~kg}$ air $/ \mathrm{kg}$ fuel (equivalence ratio $\mathrm{ER}=1.0$ ).

Table 3 shows some calculation results for the conditions of adiabatic combustion of gaseous pyrolysis products with a lack of an oxidizer.

Table 3. Parameters and composition of combustion products.

\begin{tabular}{|c|l|c|c|c|}
\hline $\begin{array}{c}\text { Parameters and } \\
\text { composition }\end{array}$ & \multicolumn{4}{|c|}{ Value } \\
\hline $\mathrm{ER}$ & 0.3 & 0.4 & 0.5 & 0.6 \\
\hline $\mathrm{T}, \mathrm{K}$ & 1166 & 1248 & 1590 & 1844 \\
\hline $\mathrm{H}_{2}$, vol. \% & 4.81 & 14.06 & 9.20 & 5.14 \\
\hline $\mathrm{CO}$, vol. \% & 30.14 & 29.71 & 23.28 & 18.01 \\
\hline $\mathrm{CH}_{4}$, vol. \% & 6.06 & 0.17 & $<10^{-6}$ & $<10^{-8}$ \\
\hline $\mathrm{N}_{2}$, vol. \% & 55.01 & 55.32 & 60.48 & 64.66 \\
\hline $\mathrm{CO}_{2}$, vol. \% & $<10^{-3}$ & 0.01 & 2.89 & 5.31 \\
\hline
\end{tabular}

Notably, at ER less than 0.3 , the concentration of acetylene and ethylene in the products of incomplete 
combustion increases. Based on the obtained results, we suggest that in order to provide thermal energy for pyrolysis processes, it is advisable to burn a part of the gases with ER values of at least 0.3.In real conditions, this ratio will depend on the intensity of heat transfer from the combustion products to the outer surface of the pyrolysis chamber and then directly to the reagents in it.

Further afterburning of products allows to obtain additional heat energy used for preheating and drying waste, as well as for heating the primary air and for other purposes. The dependence of the adiabatic combustion temperature on the equivalent ratio is shown in Fig. 2.

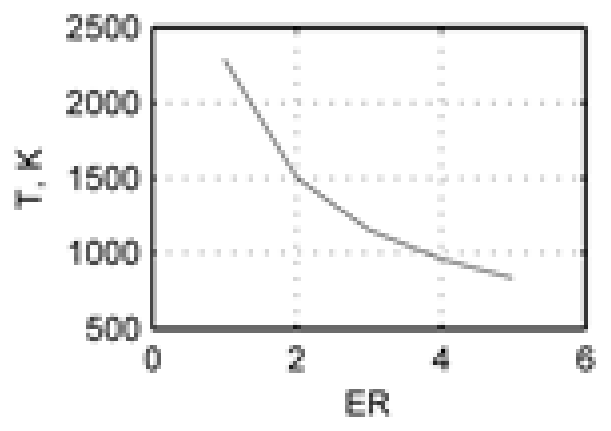

Fig. 2. Dependence of the adiabatic combustion temperature on the ER.

In after burning, the main requirement is to reduce the concentration of carbon monoxide, nitrogen oxides and unburned hydrocarbons. Therefore, after the initial incomplete combustion, secondary air should be supplied into the combustion chamber 3 (see Fig. 1) in quantities greater than stoichiometric $(E R \geq 1.6)$ and then tertiary air into the mixing chamber 4 (see Fig. 1) to achieve the required temperature level at the exit. Such organization of processes ensures the conditions needed for the complete afterburning of products and reducing the emission of nitrogen oxides.

\section{Conclusion}

Full-scale experiments were conducted on a model setup for waste pyrolysis. For a mixture of various types of carbon-containing wastes, the total yield of the liquid fraction and the gaseous non-condensable fraction was up to 93 wt. \%. The yield of the coal residue and ash was about 5 wt. $\%$ and 2 wt. \%, respectively. Heat energy obtained during incomplete combustion of about $30 \mathrm{wt}$. $\%$ gaseous products was consumed for the implementation of pyrolysis processes. Since the chemical composition of the pyrolysis products and the optimal modes of organizing the processes depend on the chemical and aggregate state of the starting materials, it is necessary to control temperature levels in the thermal decomposition chamber, combustion chamber and mixing chamber, and to regulate the air supply into these chambers.

\section{References}

1. E. Kwon, S. Kim, J. Lee, J. of $\mathrm{CO}_{2}$ Utilization, 31, 173-180 (2019)

2. J. Zeaiter, Fuel, 133, 276-180 (2014)

3. G. López, et all, Fuel, 89, 8, 1946-1952 (2010)

4. P. Williams, Waste Management, 33, 8, 17141728 (2013)

5. N. Miskolczi, et all, Fuel Processing Technology, 90 (7-8), 1032-1040 (2009)

6. S-H. Jung, et all, Fuel Processing Technology, 91, 3, 277-284 (2010)

7. H. Bockhorn, et all, J. of Analytical and Applied Pyrolysis, 49 (1-2), 53-74 (1999)

8. A. Adeniyi, et all, Nigerian J. of Technology, 37, 4, 945-949 (2018)

9. A. Osman, et all, Environ. Sci. Eu., 32, 112, 1-12 (2020)

10. A. Fivga, I. Dimitriou, Energy, 149, 865-874 (2018)

11. G. Elordi, et all, J. of Hazardous Materials, 144, 3, 708-714 (2007)

12. S. Papuga, et all, Thermal Science, 20, 731-741 (2016)

13. A. Oyedun, et all, Energy, 75, 127-135 (2014)

14. L. Sørum, et all, Fuel, 80, 9, 1217-1227 (2001)

15. V. Chhabra, et all, Waste Management, 90, 152167 (2019)

16. A. López, et all, Waste Management, 30, 4, 620627 (2010)

17. A. Demin, et all, E3S Web of Conferences, 161, 01081 (2020) 